\title{
NONLOCAL RESPONSE IN A SIMPLE EPIDEMIOLOGICAL MODEL
}

\author{
K.R. HEIDERICH ${ }^{\dagger *}$, W. HUANG ${ }^{\dagger}$, AND C. CASTILLO-CHAVEZ $^{\dagger \dagger}$
}

\begin{abstract}
We use a simple epidemiological model to investigate the effects of a nonlocal feedback on the disease dynamics. We consider a context in which two groups can be identified: the population that is experiencing an epidemic and an "outside" population that acts primarily as a source and sink of uninfected individuals. The disease dynamics of the afflicted group is affected by immigration from the outside group, which, in turn, is affected by the state of the disease. The response to this type of feedback can be investigated using a model in which the net influx of susceptibles into the afflicted population is a function of the disease prevalence in that group. We extend and generalize the analysis of Blythe et al. (1997), Brauer et al. (1998) and Hadeler and Castillo-Chavez (1995) to include more general net immigration functions. Since the population does not respond instantaneously to the state of the epidemic we include a time delay in the immigration function. We find that state-dependent recruitment, which was found to be destabilizing by Blythe et al. and Brauer et al. (1998), can actually stabilize the dynamics when such a time delay is included. Furthermore, consistent over(under)-estimation of the disease prevalence reduces(increases) the size of the endemic equilibrium populations.
\end{abstract}

Key words. Epidemiological modelling - Nonlocal response - Behaviour-change Time-delay.

1. Introduction. The mathematical modelling of epidemics plays a crucial role in understanding the dynamics of many infectious diseases (for an introductory review, see, e.g., Hethcote (1989, 2000), Brauer and Castillo-Chavez (2001), and Brauer (2001a, 2001b; this volume). Models are particularly helpful as experimental tools with which to evaluate and compare control procedures and prevention strategies, and to investigate the relative effects of various sociological and biological factors on the spread of a disease. Recent advances have been made on many fronts. For example, models help us understand the consequences of the long and variable incubation and infectivity periods of the human immunodeficiency virus (HIV), which is believed to lead to acquired immunodeficiency syndrome (AIDS) [see, e.g., Castillo-Chavez et al. (1989a,b,c,d), and Thieme et al. (1989), (1993)]. Other models are helping to identify the role of nonhomogeneous mixing in populations, [see, e.g., Busenberg et al. (1991), Castillo-Chavez et al. (1991), Hyman and Stanley (1988), Hyman, Li, and Stanley (1994), Jacquez, et. al. (1988), Sattenspiel (1987a,b) and Sattenspiel et al. (1988)] and of the dynamical response of a population to an

${ }^{*}$ Department of Physics, Langara College, 100 West 49th Avenue, Vancouver, B.C., Canada, V5Y $2 Z 6$.

${ }^{\dagger}$ Department of Mathematical Sciences, University of Alabama in Huntsville, Huntsville, AL 23599, USA

$\ddagger$ Department of Biometrics, Statistics and Theoretical and Applied Mechanics, Cornell University, 432 Warren Hall, Ithaca, N. Y. 14853-7801, USA 
epidemic [see, e.g., Blythe et al. (1993a,b, 1997), Brauer et al. (1998) and Hadeler and Castillo-Chavez 1995].

The responses of a population to the stresses of a disease are many fold, can occur at many levels, and can, in turn, significantly effect the disease dynamics. For example, governments may introduce prevention and control strategies such as vaccination schedules, education programs, and, even, mandatory quarantine [Perez-Stable (1991)]. At a more individual level, behavioural factors may come into play, which might change social interactions and the overall mixing structure, effect immigration patterns and migratory routes, and encourage more cautious and prevention-oriented behaviour. The type, degree, speed, and variety of responses depends on the particulars of both the population and the epidemic. Some factors which can influence the population's response include the severity of the epidemic, the lethality of the disease, the ease of contraction, educational awareness, public consciousness, availability of funds, and scientific knowledge. In attempts to control the disease, it is important to recognize which factors are involved and their potential impact on the disease dynamics.

When the time-scale of the epidemic is much shorter than the population's reaction time, one expects that the dynamical response of the population will have little effect on the current epidemic. However, when the time-scales are comparable, this feedback may be significant. For example, in the HIV/AIDS context, it has become clear that many behavioural changes are occurring at both the population and individual levels: the number of contacts and the number of partners are being reduced, the degree of interaction and the mixing structure is changing, [see, e.g., Centers for Disease Control (1985), Evans et al. (1989), Martin (1987), McKusick et al. (1985a,b), Griensven et al. (1989), Fineberg (1988), McClam, 2001]. Even if the effects of changing behaviour are not significant on the timescale of individual epidemics, they may have significant consequences on an evolutionary time-scale [see, e.g., Ewald (1993)].

The dynamical consequences of some behavioural responses have been investigated by a number of researchers. For example, Blythe et al. (1991) investigate the effects of disease prevalence on incidence rates. Palmer et al. (1991) study the consequences of state-dependent mixing. VelascoHernandez and Hsieh (1994) analyze a model which takes into account a change in contact rate that results from individuals realizing that they are infected. Brauer et al. (1998) investigated the effects of the proportion versus the number of infected individuals on HIV dynamics. Velasco et al. (1996) and Hsieh and Cooke (2000) look at the impact of behaviour changes in populations where treatment is available

In these models, attention is essentially restricted to "local" population responses, i.e., only the behavioral response of the afflicted group is considered. Nonlocal effects may also be important. "Outside" groups can function as sources and sinks of individuals. For example, immigrants from a group which implements a different vaccination program could seri- 
ously undermine the vaccination strategy in the population being studied. In order to take into account such nonlocal effects in general, one must understand the structures that can exist in a population.

Structure in a population can occur at many levels: groups can be distinguished by age, sex, social interactions, geographical separation, contact levels, and so on. While it can be quite difficult to identify the relevant groupings, the particular choice can significantly affect the results of the analysis. Here we briefly consider two important historical modeling examples. Sattenspiel (1987a,b) and Sattenspiel and Simon (1988) discuss the 1979 hepatitis A outbreak in some regions of New Mexico among children attending day care. Within the set of daycare centers, most of the cases occurred in a small group of family owned centers. Mixing within this group of related centers was higher than mixing within the group composed of the remaining centers and higher than mixing between the two groups. In trying to understand the spread of the disease among the children, Sattenspiel and Simon argue that it is important to recognize this two-group structuring of the population. The dynamics were essentially driven by the group of family-owned centers while the role of the remaining centers was minimal. Hethcote and York (1984) postulate the existence of two distinct groups in their study of sexually transmitted diseases (STD): a "core-group", which constitutes the most sexually active group in the sexually-active population, and the "meta-population", which encompasses the rest of the sexually-active population. They found that most new cases of STD were generated by the core group. Again, the firstorder dynamics were essentially driven by the core group and the effect of the meta-population was less important.

Theses examples motivate us to consider an epidemiological context in which essentially two groups can be identified: the core group and the outside population. The afflicted group can be regarded as driving the disease dynamics, while the coupling between groups, as well as the disease dynamics within the outside group, can be regarded as secondary. This type of division occurs naturally in many situations as illustrated by the preceding examples. The groupings are not static due to migration into and departure from each group, which most models have not taken into consideration [but see Scalia-Tomba (1991), Blythe et al. (1993b), Hadeler and CastilloChavez (1995)]. Net immigration between the outside population and the afflicted group may influence the dynamics of the epidemic. The immigration may, in turn, depend on the state of the disease in the "target" group (Fineberg (1988)). The consequences of some state-dependent recruitment scenarios have been investigated by Blythe et al. (1993b), Brauer et al. (1998), and Hadeler et al. (1995). Blythe et al. (1997) and Brauer et al. (1998) consider a simple two-variable $(S I)$ epidemiological model. In that model, the equilibria are stable if the recruitment into the afflicted group is constant. However, if net immigration drops off as a function of the disease prevalence in the target population, then the endemic equilibrium 
can be destabilized. Brauer et al. (1998) considered the case where information on prevalence was available either in total numbers or as a fraction. Hadeler and Castillo-Chavez (1995) considered the role of education and the size of the non-core population (a dynamic variable). Hadeler and Castillo-Chavez' (1995) simple model is capable of supporting multiple endemic equilibria whenever $R_{0}$, the basic reproductive number, is less than one. This possibility had been previously illustrated by Castillo-Chavez et al. (1989b) and Huang et al. (1992) but in a more complex setting. It had been shown that the assumption of asymmetric probabilities of transmission between groups could generate multiple endemic equilibria via a backward bifurcation.

It is this "behavioural" response of the population at large to the population being studied that we investigate in detail using more realistic immigration functions in order to clarify the mechanisms relevant to the dynamics. In Blythe et al.'s or Brauer et al.'s models, the population is assumed to have instantaneous knowledge of the state of the epidemic. The response will be time-lagged, in general, due to the fact that it takes time to assess the state of the epidemic, time to publicize the information, and time for the population to react to the information. We will show that the inclusion of a discrete time delay in the immigration term can actually stabilize the dynamics.

We also deal explicitly with aspects of the fact that one never has exact knowledge of the state of the epidemic. Although exact knowledge is often assumed (e.g., Blythe et al. (1997); Brauer et al. (1998); Hadeler and Castillo-Chavez (1995)), there will always be a degree of inaccuracy and uncertainty. For example, the prevalence may be over- or under-reported, its severity over- or underestimated. It is the perceived state of the epidemic, not the actual state, to which the population will respond. If we suppose that the prevalence figures are consistently deflated or inflated by a constant fraction, or that the population, on average, consistently misinterprets the reports, then we find that if the figures are over-estimated then the size of the endemic equilibrium population is lower and, conversely, if they are under-estimated then the equilibrium size is higher. Although the parameter ranges for stability are slightly changed, the dynamics are qualitatively the same. This underscores the influence of the media and the value of educational programs.

In the next section, we investigate the dynamical consequences of a general state-dependent net immigration function on the disease dynamics of a simple epidemiological model. After discussing the model assumptions, we consider three functional dependencies of the immigration term, give the disease-free and endemic equilibria and their stability. In Section III, we study the effects of a time delay in the functional dependencies of the immigration term. Section IV contains a brief summary of our results. 
2. State-dependent immigration model. As discussed in the introduction, we consider epidemiological contexts in which essentially two groups can be identified: the population which is experiencing an epidemic and the outside population. The outside population is weakly coupled to the afflicted group and its primary effect is as a source and as a sink of individuals to and from the afflicted population. The epidemic is driven by the outbreak in the afflicted group while the effect of the outside population is purely secondary and can be neglected as a higher order effect. Hence, in what follows, we consider the dynamics of only one group, namely the afflicted group. The number of healthy individuals in that group are called susceptibles and are denoted by $S(t)$, and the number of infected individuals, by $I(t)$, where $t$ denotes time. To a first approximation, the progress of the disease can be modelled by two first-order ordinary differential equations,

$$
\begin{aligned}
& \dot{S}=g-\mu S-f, \\
& \dot{I}=f-\alpha I,
\end{aligned}
$$

where the recruitment function, $g=g(S, I)$, describes net immigration, $\mu$ is the (constant) natural mortality, the force of infection term, $f=f(S, I)$, describes the contact structure between infected and susceptible individuals, $\alpha=\sigma+\mu$, where $\sigma$ is the constant migration out of the infected class due to death, recovery, emigration, etc., and the dot denotes derivative with respect to time. In this simple system there are four (or more) parameters (depending on the form of $g$ and $f$ ). $\sigma$ and $\mu$ have the dimensions of inverse time, and the functions $g$ and $f$ have the dimensions of number of individuals per unit time. To simplify the analysis, we do not include a recovered class. Thus, we implicitly assume that the disease is fatal, that all recovered individuals leave the afflicted group, or that the epidemic is short-lived relative to the length of illness and, therefore, that the recovered class does not contribute significantly to the dynamics. Since we are primarily interested in the effects of state-dependent net immigration, we do not include additional complications such as incubation periods. Given the context of interest, we do not include a birth term, although this would be straightforward to do. We also assume that immigrants are not yet infected [but see Hoover et al. (1991)].

The form of the force of infection, $f=f(S, I)$ depends on the particulars of the disease. Here we take the general form

$$
f=c \frac{S I}{S+I},
$$

where $c$ is a constant having the dimensions of inverse time. This term needs some justification (see also Busenberg and Cooke (1993)): suppose that a susceptible has $x$ contacts per unit time. Assuming homogeneous mixing among susceptibles and infecteds, a fraction $I /(S+I)$ of these 
contacts are with infected individuals. (This assumes that all of the infecteds are intermingling as freely as those uninfected, i.e., that the disease is not sufficiently debilitating to restrict the movement of infecteds (which is a good approximation for some diseases such as gonorrhea and syphilis). More generally one could take into account the reduced mobility of infecteds by setting the fraction of contacts with infecteds to be $\rho I /(S+\rho I)$ where $\rho$ represents the degree of non-homogeneity in the mixing (e.g., $\rho \ll 1$ for diseases which immobilize those infected and thereby significantly reduce the mixing of susceptibles with infecteds)). In the case of models for sexually-transmitted HIV, it is often assumed that individuals with AIDS are sexually-inactive (Castillo-Chavez et al. 1989a). Let the probability of contracting the disease per contact be $p$. Then the total number of contacts per unit time which lead to infection is given by Eq. (2) with $c=x p$. For some diseases it is reasonable to assume that the number of contacts per unit time, $x$, is a function of the total population size, $N=S+I$, i.e., $x=x(N)$. Additionally, one frequently assumes that $x(N)$ is directly proportional to $N$ - this gives the so-called mass-action force of infection term $f=\beta S I$, where $\beta$ is a constant. It is unlikely, however, in most epidemiological contexts that the number of contacts per unit time will increase indefinitely with population size. Therefore it is appropriate to introduce an upper threshold to the possible number of contacts per unit time. Then the force of infection term becomes $f=c(N) S I /(S+I)$, where $c(N)$ is an increasing function of population size assymptoting to the upper threshold at large $N$, e.g., $c(N)=c_{0} N /(1+N)$, (c.f. Holling's type II functional response, Holling (1959)). Since we take $c(N)=c=$ constant, we are therefore assuming that the changing size of the afflicted group does not significantly effect the contact rate. If we are considering a population that is distinguished by its (high) contact level (near the upper threshold, say, e.g., the "core" group in STD), then taking $c$ to be a constant is appropriate: the group being modelled is near its contact threshold, in fact, it is defined by its contact level $c$. If the contact level of an individual changes so that the mixing is no longer described for that individual by the parameter $c$, then that individual is considered to have left the group. This is taken into account by the net immigration function, $g=g(S, I)$, and the emigration term, $\sigma$. Note, however, that since $c$ is constant, we are assuming that the total population size in the afflicted group remains high enough to sustain the high contact level. Alternatively, if we are considering a population distinguished by, say, geographic location, the assumption that the parameter $c$ is constant means that there is not much that this population does, or can do, to change their contact rate. Our prior work on HIV (Castillo-Chavez et al. 1989a) has shown that there is no qualitative difference between constant $c$ and some realistic functional forms for $C(N)$.

There will generally be some mixing between the afflicted group and the outside population. We assume that this is a higher order effect that 
can be ignored. Explicitly, inter-group mixing could be included by taking the force of infection term to be, say,

$$
f=\left(x_{A} \frac{I_{A}}{S_{A}+I_{A}}+x_{B} \frac{I_{B}}{S_{B}+I_{B}}\right) p S_{B}
$$

where the subscript $A$ refers to the outside population which we call here group $A$, and the subscript $B$, refers to the afflicted population, here group $B$. A susceptible in group $B$ will have $x_{B}$ contacts per unit time with members of group $B$, and $x_{A}$ contacts per unit time with members of group $A$. By assumption, there is little mixing between groups so that $x_{B} \gg x_{A}$. Further, we are interested in situations when an epidemic is occurring in group $B$ and, although there are infections occurring in group $A$, the level of infection is such that $I_{A} / N_{A} \ll I_{B} / N_{B}$, where $N_{A}=S_{A}+I_{A}$, etc.. Combining inequalities, we have that $x_{A} I_{A} / N_{A} \ll x_{B} I_{B} / N_{B}$, and hence can neglect the second term in Eq. (3). Of course, $I_{B}$ and $S_{B}$ are dynamical quantities so that this condition should be satisfied throughout the course of the epidemic. In the absence of disease in group $B$ we assume, of course, that there is no disease in group $A$.

It remains to discuss plausible forms for the immigration function $g=g(S, I)$. We assume that as the disease prevalence increases, net immigration into the susceptible population decreases. We further assume that the state of the epidemic is reported to the population in terms of either (i) the fraction of infecteds, $\eta=I /(S+I)$, so that $g=g(\eta)$, or (ii), the actual numbers of infected individuals, $I$, so that $g=g(I)$. Motivated by Blythe et al., Brauer et al. and Hadeler and Castillo-Chavez (1995), we consider the following general class of decreasing functions:

$$
g(\xi)=a e^{-b \xi^{\gamma}},
$$

where $\xi$ is taken to be (i) $\eta$, or (ii) $I$, and where $a, b$ and $\gamma$ are real positive numbers. The dimensionless parameter $\gamma$ is the shape parameter of the response function $\exp \left(-b \eta^{\gamma}\right)$ and describes the rate of reaction of the population: Small $\gamma(<0.5)$, describes a response curve that drops off sharply as a function of infection (i.e., $g(\eta)$ convex), while, larger $\gamma(>3)$, is appropriate to describe the response of a population that is more gradual (i.e., $g(\eta)$ concave). The parameter $b$ is related to the scaling parameter, $b^{-1 / \gamma}$, and describes the degree of the reaction: As $b$ decreases in value, the reduction in net immigration will be less severe. The functional form given in Eq. (4) is quite general and describes a wide variety of plausible responses. Note that the function (4) is derived from the Weibull distribution and is often used to model survivorship curves in demographic models.

It would also make sense to consider other functional dependencies of $g=g(S, I)$. In the immigration function given by Eq. (4), we are assuming that the population is reacting to the precise state of the epidemic. However, assessments of the state of the epidemic will rarely be so accurate. 
To get a rough first approximation of the effects of misinformation, we assume that the population consistently over- or under-estimates the disease prevalence by a constant proportion. This leads one to consider immigration functions that depend on, case (iii), $\tilde{\eta}=\chi I /(S+\chi I)$, where $\chi$ is a constant parameter approximating the degree of over- $(\chi>1)$ or underestimation $(\chi<1)$ of the disease prevalence, (more generally, $\chi$ could be a random variable). Taking a recruitment function of the form given in Eq. (4) with functional dependence $g=g(\tilde{\eta})$, one finds qualitatively the same dynamics as in case (i). However, the parameter range of the various dynamics depends on $\chi$. Additionally, it may be appropriate to take the immigration function $g$ to be a decreasing function of the real or perceived virulence, $g=g(\nu)$. Such a model could be used to compare the relative importance of the recruitment term for different diseases which are distinguished by their virulence, however, we do not investigate this possibility here.

In summary, to model the disease dynamics (in the afflicted group), we take the system given by Eqs. (1) together with the form of the force of infection function, Eq. (2), and the form of the immigration function, Eq. (4), with functional dependencies (i) $g(\eta)$, (ii) $g(I)$, and (iii) $g(\tilde{\eta})$. For case (i), the system can be written, in dimensionless units, as follows

$$
\begin{aligned}
& \dot{S}=a e^{-b \eta^{\gamma}}-\mu S-\frac{I S}{(I+S)}, \\
& \dot{I}=\frac{I S}{I+S}-\alpha I
\end{aligned}
$$

where we have non-dimensionalized by setting $S \rightarrow S K, I \rightarrow I K, c t \rightarrow t$, $a \rightarrow a K c, \mu \rightarrow \mu c, \sigma \rightarrow \sigma c$, and $c=1$, where $K$ is some constant reference population level. The parameter $c$ has been eliminated and there are only four remaining (dimensionless) parameters $\mu, \sigma, a$, and $b$.

First, let us consider case (i) where recruitment is taken to be a function of the fraction of infecteds, $g(\eta)=a \exp \left(-b \eta^{\gamma}\right)$, Eq. (4). The diseasefree equilibrium of the system (5) is

$$
\bar{S}_{0}=\frac{a}{\mu}, \quad \bar{I}_{0}=0 .
$$

This equilibrium, $\left(\bar{S}_{0}, \bar{I}_{0}\right)$, is globally stable if $1<\alpha$ (see Appendix A). If $1>\alpha$, the endemic equilibrium of the system (5) is

$$
S_{0}=g_{0} \frac{1}{1-\sigma} \quad, \quad I_{0}=S_{0} \frac{1-\alpha}{\alpha},
$$

where $g_{0}=g\left(\eta_{0}\right)$ and from Eqs. (5) one has that $\eta_{0}=1-\alpha$. The size of the endemic equilibrium population $\left(S_{0}, I_{0}\right)$ increases as $\gamma$ increases: For larger $\gamma$, immigration will be relatively high at a given incidence level as compared to a population response that is described by smaller $\gamma$. The endemic equilibrium, $\left(S_{0}, I_{0}\right)$, Eq. (7), is stable provided that

$$
\partial_{S} g_{0}<1-\sigma
$$


where $\partial_{S}=\partial / \partial S$ and $\partial_{S} g_{0}$ denotes $\left.\left(\partial_{S} g\right)\right|_{0}=\left.\left(\partial_{S} g\right)\right|_{\left(S_{0}, I_{0}\right)}$. For the special case $\gamma=1$, it can be shown that the equilibrium $(7)$ is globally stable (Huang, personal communication), if (8) holds. At $\partial_{S} g_{0}=1-\sigma$, the characteristic equation associated with the endemic equilibrium has a pair of purely imaginary roots and we have a Hopf bifurcation.

Substituting in $g(\eta)=a \exp \left(-b \eta^{\gamma}\right)$, the stability condition (8) can be written

$$
\text { b } \alpha \alpha(1-\alpha)^{\gamma}<1 .
$$

Figure 1 shows the regions of stability in terms of the parameters $b$ and $\alpha$. As $\gamma$ increases, the boundary between the stable and unstable regions shifts towards smaller $\alpha$. In other words, if the response of the population is gradual (large $\gamma$ ), the system will be stable for smaller values of $\sigma$, lower emigration. Conversely, for small $\gamma$, i.e., for a rapid response, the system will be unstable except for $\alpha$ large or small. Varying the parameter $\mu$ gives qualitatively similar curves as in Figure 1, however, the shape of the curves are modified

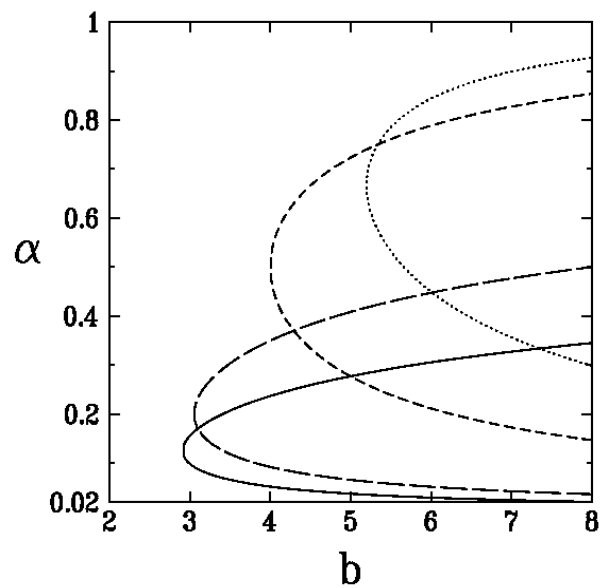

FIG. 1. Stability regions of the endemic equilibrium (7) for the system (5) with $g=a \exp \left(-b \eta^{\gamma}\right)$, case (i), for four different $\gamma: \gamma=0.5$ (dotted line), $\gamma=1.0$ (shortdashed line), $\gamma=4.0$ (long-dashed line), and $\gamma=7.0$ (solid line). Each curve satisfies $E q$. (9) with $a=0.02$ and $\mu=0.02$. To the left of each curve, the endemic equilibrium is stable, while to the right, it is unstable. We choose values of a and $\mu$ appropriate for gonorrhea (Hethcote and Yorke (1984)).

For the case $\gamma=1$, the equilibrium (7) is globally stable if $b \alpha(1-$ $\alpha)<1$. Thus, in terms of the parameters $b$ and $\alpha$, we have stability under the following conditions: if $b<4$, for all $\alpha$; if $b=4$, except when $\alpha=1 / 2$; if $b>4$, for $\alpha>\alpha_{+}$or $\alpha<\alpha_{-}$, where $\alpha_{ \pm}$are the roots of $b \alpha(1-\alpha)=1$. Recall that the parameter $b$ describes the degree of reaction 
of the population and the parameter $\alpha=\mu+\sigma$, where $\sigma$ describes the migration out of the infected class. These results are essentially equivalent to those in Blythe et al., where the model (5) is studied with $\gamma=1$.

It will prove conceptually helpful in the next section to show how the local stability condition, $\partial_{S} g_{0}<1-\sigma$, Eq. (8), arises from a second-order ordinary differential equation. Linearizing system (5) about the endemic equilibrium, $\left(S_{0}, I_{0}\right)$, one gets two first-order ordinary differential equations for the linear perturbations $S_{1}$ and $I_{1}$, (i.e., $S=S_{0}+\epsilon S_{1}$ and $I=I_{0}+\epsilon I_{1}$, for $\epsilon$ small). Combining the equations for $\dot{S}_{1}$ and $\dot{I}_{1}$, one gets the secondorder equation for $S_{1}$ :

$$
\ddot{S}_{1}+(A-C) \dot{S}_{1}+(B+D) S_{1}=0
$$

where

$$
\begin{aligned}
& A=\partial_{S} f_{0}+\mu+\alpha-\partial_{I} f_{0} \\
& B=\alpha\left(\partial_{S} f_{0}+\mu\right)-\mu \partial_{I} f_{0}, \\
& C=\partial_{S} g_{0} \\
& D=\partial_{S} g_{0}\left(\partial_{I} f_{0}-\alpha\right)-\partial_{I} g_{0} \partial_{S} f_{0} .
\end{aligned}
$$

Equation (10) is the equation for a damped harmonic oscillator. Since linear oscillators are well understood, the conditions for stability can be read directly from Eq. (10): For negative restoring force and positive damping, $B+D>0$, and $A-C>0$ respectively, the system is damped so that the equilibrium is stable. On the other hand, if $A-C<0$, the oscillations are unstable. This is (of course) precisely condition (8). For the recruitment function $g=a \exp \left(-b \eta^{\gamma}\right)$ and a force of infection term $f$ given by Eq. (2), at the endemic equilibrium (7), we have $A=1-\sigma, B=A \alpha(1-\alpha)$, $C=A \alpha b \gamma(1-\alpha)^{\gamma}$, and $D=0$. Thus, one sees that the condition $A-C>0$ is precisely the stability condition given by Eq. (9). (Of course, this is simply the Routh-Hurwitz criteria in two dimensions (see, e.g., Murray (1990)), where the determinant of the eigenvalue matrix of the linearized system is $B+D>0$ and the trace, $-(A-C)<0$. The harmonic oscillator analogy is, however, conceptually useful.)

These results are quite different than those obtained when the effect of the disease on net immigration is not taken into account. In this case, the recruitment is constant, which corresponds to taking $b=0$ in Eq. (4) (giving $g(S, I)=a)$. The disease-free and endemic equilibria are again given by Eqs. (6) and (7) respectively. However, for $b=0$, the endemic equilibrium is always stable since the stability condition, $\partial_{S} g_{0}=0<1-\sigma$, Eq. (8) always holds. On the other hand, if there is state-dependent immigration that is appropriately described by the function $g(\eta)=a \exp \left(-b \eta^{\gamma}\right)$, the system is unstable when $\partial_{S} g_{0}>1-\sigma$. Further, due to the reduced net immigration as the epidemic spreads, the size of the endemic equilibrium population is smaller for nonzero $b$. 
Now, we consider case (ii), where the immigration term is taken to be a function of the number of infecteds, $g(I)=a \exp \left(-b I^{\gamma}\right)$. The equilibria of the system (5) with this functional dependence of the recruitment function are the same as in case (i) and are given by Eqs. (6) and (7). However, in this case the endemic equilibrium, $\left(S_{0}, I_{0}\right)$, is only defined implicitly by Eqs. (7) since $g_{0}=a \exp \left[-(1-\alpha)^{\gamma} S_{0}^{\gamma}\right]$. The stability conditions are also as in case (i): the disease-free equilibrium is stable for $1<\alpha$ and the endemic equilibrium is stable for $\partial_{S} g_{0}<1-\sigma$, Eq. (8), i.e., $C<A$. For $g(I)=$ $a \exp \left(-b I^{\gamma}\right)$, the coefficients $A$ and $B$ in (10) are as before. However, from (13) and (14) one finds that $C=\partial_{S} g_{0}=0$, and that $D=-\partial_{I} g_{0}(1-\alpha)^{2}$. Thus, the stability condition given by Eq. (8), $\partial_{S} g_{0}=0<1-\sigma$, is trivially satisfied, and the endemic equilibrium for system (5), with the recruitment function $g(I)=a \exp \left(-b I^{\gamma}\right)$, is always stable (in fact, globally stable, by the Bendixson-du Lac criterion, see, e.g., Clark (1990)). Consequently, if the state-dependent recruitment is best described as a function of $I$ of the form (4), then, the equilibria are stable although the size of the equilibrium population is smaller than for constant recruitment.

As a final functional dependency of the immigration function, we consider case (iii), $g=g(\tilde{\eta})$, which crudely includes the effects of misinformation. Rather than taking recruitment to depend on the precise ratio of infecteds, suppose that the recruitment depends on $\tilde{\eta}=\chi I /(S+\chi I)$, where $\chi$ is a (dimensionless) positive real parameter that accounts for the fixed degree of over- or under-estimation of the disease prevalence on the part of the population. As one would expect, over-estimation, $\chi>1$, leads to smaller endemic equilibrium populations, i.e., $\tilde{S}_{0}<S_{0}$, while underestimation, $\chi<1$, leads to higher equilibrium populations, i.e., $\tilde{S}_{0}>S_{0}$, where $\tilde{S}_{0}$ is the equilibrium size of the susceptible population as determined from the recruitment function $g(\tilde{\eta})$, where $\tilde{g}_{0}=g\left(\tilde{\eta}_{0}\right)$ with $\tilde{\eta}_{0}=\chi(1-\alpha) /[\alpha+\chi(1-\alpha)]$, i.e., $\tilde{S}_{0}=\tilde{g}_{0} /(1-\sigma)$, as in Eq. (7). For $g(\tilde{\eta})$, the coefficients $A, B, D$ of the linearized system (10) are as for $g(\eta)$ and are given by Eqs. (11), (12), and (14). However, $C=\partial_{S} g_{0}$ is slightly modified by the parameter $\chi$ to

$$
C=A \alpha b \gamma \chi^{\gamma} \frac{(1-\alpha)^{\gamma}}{(\alpha+\chi(1-\alpha))^{\gamma+1}} .
$$

By inspection of the "harmonic oscillator" equation for $S_{1}$, Eq. (10), one sees that qualitatively, the dynamics of the system (5) will not be different than those with $\chi=1$. Indeed, in the next section we combine cases (i) and (iii). Figure 2 shows how the boundary between stable and unstable regions, in terms of $b$ and $\alpha$, is modified by $\chi$ : Overestimation of the disease prevalence, i.e., $\chi>1$, shifts the curve to higher $\alpha$, while underestimation, shifts the curves to lower $\alpha$. Figure 3 is a phase-plane diagram for a parameter choice where the endemic equilibrium is unstable. Note that the size of the population can change by almost an order of magnitude. Recall from Section 1, that since we take the contact rate, $c$, to be constant, we are assuming that the size of the population remains large enough to sus- 
tain this contact rate. Although we will not do so in this paper, one might also consider a contact rate that depends on the size of the population, $N$, e.g., $c(N)=c_{0} N /(1+N)$, since $N$ can change dramatically. In the next section, we will see how a time delay affects the results of this section.

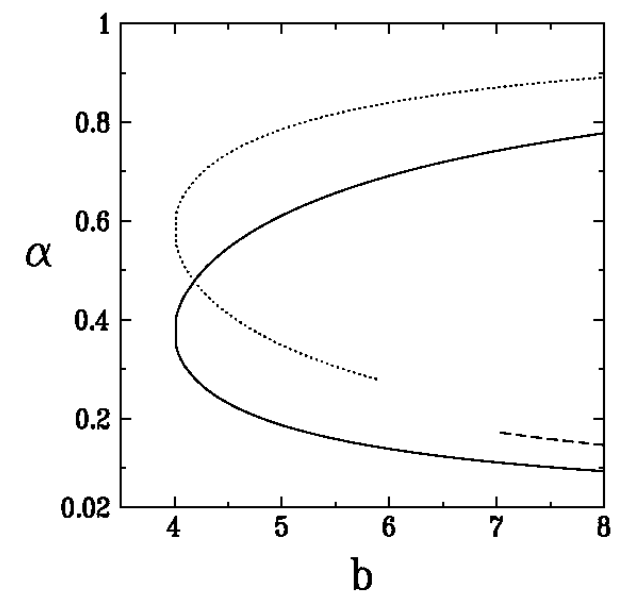

FIG. 2. Stability regions of the endemic equilibrium (7) for the system (5) with $g=a \exp \left(-b \tilde{\eta}^{\gamma}\right)$, case (iii), for three different $\chi: \chi=0.6$ (solid line), $\chi=1.0$ (shortdashed line), and $\chi=1.4$ (dotted line). The curves are $A=C$, where $A=c-\sigma$, and $C$ is given by $E q$. (15), with $a=0.02, \mu=0.02, \gamma=1.0$. To the left of each curve, the endemic equilibrium is stable, while to the right, it is unstable.

3. Time-delayed model. The results of the preceding section motivate consideration of more realistic forms for the recruitment function. Since the information of the state of the epidemic will not be instantaneously available, the population, is, in effect, responding to the state of the epidemic at some earlier time, $(t-\tau)$. This naturally leads one to consider the effects of a discrete time delay in the recruitment term. In this section we investigate the following time delayed modification of Eq. (5):

$$
\begin{aligned}
& \dot{S}=g(\eta(t-\tau))-\mu S-f, \\
& \dot{I}=f-\alpha I,
\end{aligned}
$$

where $\tau$ is a discrete time delay, the force of infection term $f=f(S, I)$ is given by Eq. (2) and the functional form of $g=g(\xi(t-\tau))$ is given by Eq. (4). Recall that all quantities are dimensionless. We consider two cases: (i) net immigration is a function of the fraction of infecteds with $g(\tilde{\eta}(t-\tau))=a e^{-b \tilde{\eta}(t-\tau)^{\gamma}}$, where $\tilde{\eta}=\chi I /(\chi I+S)$, and case (ii), recruitment is a function of the number of infecteds, $g(I(t-\tau))=a e^{-b I(t-\tau)^{\gamma}}$. In case (i), we find that the time delay can stabilize the dynamics. Conversely, in case (ii), we find that the time delay can destabilize the dynamics. 


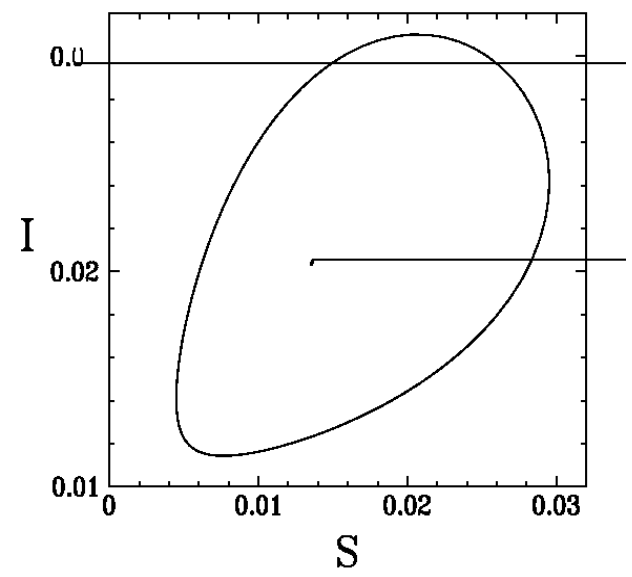

FIG. 3. Phase-plane trajectories of system (16) for case (i), $g(\tilde{\eta}(t-\tau))$. The limit cycle (counter-clockwise flow) corresponds to $\tau=0$, and the inward spiral corresponds to $\tau=5$. The parameter values are $\alpha=0.4, b=4, a=0.02, \mu=0.02, \gamma=3.0$, $\chi=1.0, \tau=5$, and are chosen to be in the range where a time delay is stabilizing. For this parameter range, $S_{0}=0.0136$ and $I_{0}=0.0204$.

While the equilibria of (16) do not depend on $\tau$, their stability does. We find that the disease-free equilibrium, $\left(\bar{S}_{0}, \bar{I}_{0}\right)=(a / \mu, 0)$, Eq. (6), is globally stable provided that $1<\alpha$ (see Appendix A). The local stability of the endemic equilibrium, $\left(S_{0}, I_{0}\right)$, of $(16)$ can be determined from the sign of the real part of the eigenvalues, $\lambda$, of the characteristic equation:

$$
0=\lambda^{2}+A \lambda+B+(-C \lambda+D) e^{-\lambda \tau},
$$

where, $A, B, C, D$ are as defined in Eqs. (11-14). For $\tau=0$, the stability condition of the non-time-delayed equilibrium is recovered (stable if $A>C$, Eq. (8). For nonzero $\tau$, there are infinitely many solutions $\lambda=\lambda(\tau)$ to Eq. (17). The endemic equilibrium $\left(S_{0}, I_{0}\right)$ of system (16) is stable when none of these roots has a positive real part. See Appendix B for an outline of the analysis for the following results: If $A>C$, a time delay has no effect on the stability of the equilibria. Since in this parameter range, the endemic equilibrium is stable for $\tau=0$, it is stable for all $\tau$. The endemic equilibrium $\left(S_{0}, I_{0}\right)$ of system (16) is said to be "absolutely stable" (Brauer (1987)) for $A>C$.

Since for $A<C$, the equilibrium is unstable for $\tau=0$, a nonzero $\tau$ may actually stabilize the dynamics in this parameter range. The $\tau$ 's for which the system is on the boundary between stable and unstable regions are defined by $\tau_{1}^{(n)}=\left(\theta_{1}+2 \pi n\right) / \omega_{1}$, and $\tau_{2}^{(n)}=\left(\theta_{2}+2 \pi n\right) / \omega_{2}$, where $n$ is a natural number, $\theta_{1}=\arcsin (A / C), \theta_{2}=2 \pi-\theta_{1}$, and $\omega_{1}=\omega_{-}, \omega_{2}=$ $\omega_{+}$, with $\omega_{ \pm}=\left( \pm \sqrt{C^{2}-A^{2}}+\sqrt{C^{2}-A^{2}+4 B}\right) / 2$, (see Appendix II for 
details). For $\tau$ between $\tau_{1}^{(0)}$ and $\tau_{2}^{(0)}$, the equilibrium is stable if $\tau_{1}^{(0)}<\tau_{2}^{(0)}$. Similarly, for $\tau$ between $\tau_{1}^{(1)}$ and $\tau_{2}^{(1)}$, the equilibrium is stable if $\tau_{1}^{(1)}<\tau_{2}^{(1)}$. These alternating regions of stability and instability (USU regions, i.e., Unstable-Stable-Unstable), will continue until $\tau_{1}^{(n+1)}>\tau_{2}^{(n+1)}$, for some $n$. In some parameter ranges, $n$ can be quite large, (e.g., for $b=12.8854$, $\alpha=0.7, \mu=0.02, \chi=1.0$, and $\gamma=1.0$, we find that $n=338$ ). The timedelay which results in stable dynamics can also be quite large (e.g., for the parameters given above, we find that $\tau_{2}^{(338)}=8.2 \times 10^{3}$, in dimensionless units. (To get the dimensionfull units, divide by the dimensionfull c. E.g., for the parameter range that is appropriate for gonorrhea (Hethcote and Yorke (1984)), $c=0.05$ days $^{-1}$ so that $\tau_{2}^{(228)}=1.6 \times 10^{4}$ days).) Figure 3 shows the phase-plane trajectories of the system (16) with $\tau=0$ and $\tau=5$, where the parameter range is chosen so that the endemic equilibrium is unstable for $\tau=0$ and asymptotically stable for $\tau=5$. The regions where a time delay may give rise to stable equilibria are shown in Figure (4a) for a representative parameter range. The time delays associated with these regions of alternating stability and instability are shown in Figure (4b). Note that in certain parameter ranges, stability is very sensitive to time delays. Figure (4b) has the characteristic "pine-tree" shape (see MacDonald (1989)). The lower part of the "branches" correspond to the $\tau_{1}^{(n)}$ 's while the upper part of the "branches" correspond to the $\tau_{2}^{(n)}$ 's. The number of oscillations can exceed $n=1599$, corresponding to time delays of over $2.6 \times 10^{4}(b=3.5007, \alpha=3.98, a=0.02, \mu=0.02, \chi=1.0$, and $\gamma=1.0$ ). Our ability to detect high $n$ is limited by the numerical mesh with which we look. Thus, while extremely high $n$ and $\tau$ are possible, the parameter range in which they occur can be vanishingly small (see Figure (4a)).

It is interesting that a finite time delay can have a stabilizing effect on the dynamics. Some insight can be obtained by writing the linearized equations as in Eq. (10):

$$
\ddot{S}_{1}+A \dot{S}_{1}+B S_{1}-C \dot{S}_{1}(t-\tau)+D S_{1}(t-\tau)=0 .
$$

For case (i), $D=0$, so that the time delay only appears in the damping term $\left(C \dot{S}_{1}\right)$. It is now intuitively plausible that if the damping is in phase with the oscillations, the system may be stabilized.

Now let us consider case (ii), where the recruitment is a function of $I, g=g(I(t-\tau))$. The first of the Eqs. (16) then becomes $\dot{S}=g(I(t-$ $\tau))-\mu S-f$. The disease-free equilibrium, $\left(\bar{S}_{0}, \bar{I}_{0}\right)=(a / \mu, 0)$, Eq. (6), is stable provided that $1<\alpha$. For the endemic equilibrium, referring to (18), one sees that the time delay in this example appears in the restoring force, $\left(D S_{1}\right)$, since for $g=g(I), C=0$, but $D \neq 0$. There are three possible stability consequences (see Appendix 2 for details): (a) If the restoring force is large enough, i.e., if $D>B$, the endemic equilibrium will be destabilized for large enough $\tau$. In this parameter range, for $\tau<\tau_{c}=$ 
(a)

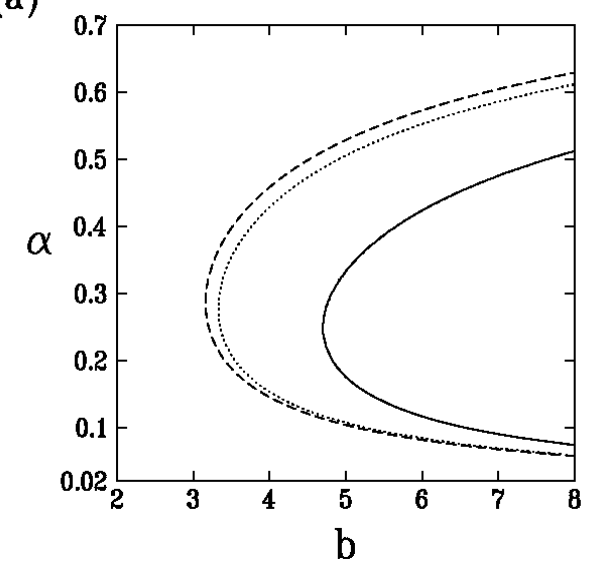

(b)

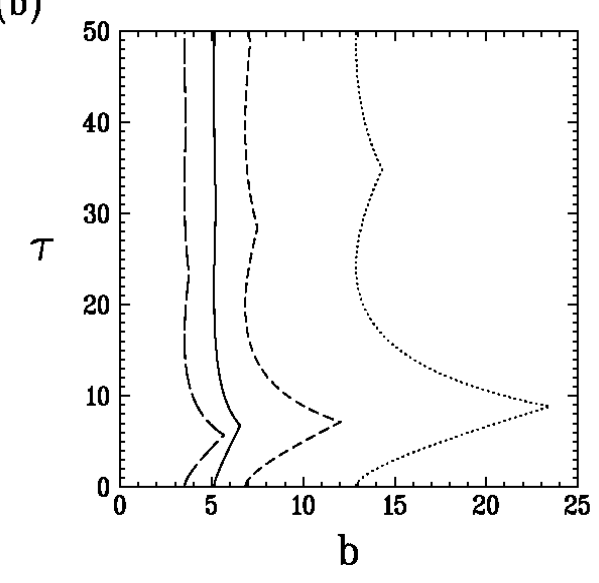

FIG. 4. (a) Stability regions of the endemic equilibrium (7) for the system (16) with $g=a \exp \left(-b \tilde{\eta}(t-\tau)^{\gamma}\right)$, and $a=0.02, \mu=0.02, \chi=1.2$, and $\gamma=3.0$. The shortdashed curve corresponds to the stability boundary for the original $\tau=0$ equations (5) (same as the short-dashed curves in Figures 1 and 2). The solid line is the boundary where $\tau_{1}^{(0)}=\tau_{2}^{(0)}$ so that to the left of this curve, the system (modelotd) is stable for certain ranges of the parameter $\tau$ (see Figure $4 b$ for the $\tau$ ranges). The dotted line is the boundary where $\tau_{1}^{(1)}=\tau_{2}^{(1)}$ so that in the region between the dashed and dotted curve, the system alternates at least twice between stability and instability as $\tau$ increases. (b) Stability regions of the endemic equilibrium (7) for the system (16) with $g=g(\tilde{\eta}(t-$ $\tau)$ ) in the $(\tau-b)$-plane for four different $\alpha: \alpha=0.1$ (solid line), $\alpha=0.4$ (long-dashed line), $\alpha=0.6$ (short-dashed line), and $\alpha=0.7$ (dotted line). The parameters are $a=0.02, \mu=0.02, \chi=1.2$, and $\gamma=3.0$. To the left of each curve, the system is stable. For $\tau=0$, model (5), the corresponding regions of stability are given approximately by: $b<3.513$ for $\alpha=0.4, b<5.13$ for $\alpha=0.1, b<6.53$ for $\alpha=0.6$, and $b<12.89$ for $\alpha=0.7$. 
$\arccos \left[\left(\omega^{2}-B\right) / d\right] / \omega$, the endemic equilibrium will be stable. However, for $\tau>\tau_{c}$, the equilibrium will be unstable ( $S U$ regions, i.e., Stable-Unstable). Case (b): If the restoring force is of intermediate range, i.e., if $B>D$ and if $\left(A^{2}-2 B\right)^{2}-4\left(B^{2}-D^{2}\right)>0$, one finds alternating regions of stability and instability provided that $2 B>A^{2}$ (SUS regions). Case (c): if the restoring force is small, $B>D$ and $\left(A^{2}-2 B\right)^{2}-4\left(B^{2}-D^{2}\right)<0$, or $2 B<A^{2}$, there will be no stability change and the endemic equilibrium will be absolutely stable. These results are summarized in Figure (5a), which shows the regions in parameter space corresponding to the various stability regimes. The parameter range where the regions of stability and instability alternate $(S U S)$ is very small and occurs for very large $b$ and very small $\mu$. (Of course, $b$, here, is dimensionless. Since the scaling parameter in Eq. (4) is actually $b^{-1 / \gamma}$, the parameter $b$ simply appears to be "unnaturally" large.) Figure (5b) shows the time delays that are associated with the various stability regions. For small $b$, instability occurs only for large $\tau$ (e.g., for $b=4$, $\alpha=0.15, a=0.02, \mu=0.001, \chi=1.0$, and $\gamma=1.0$, instability sets in when $\tau>171)$. On the other hand, for large $b$, instability can occur for quite small time delays (e.g., for $b=100, \alpha=0.4, \mu=0.001, \chi=1.0$, and $\gamma=1.0$, instability sets in when $\tau>6$ ). It might be interesting to compare these results with those obtained from a periodic forcing of the epidemiological model (1). Seasonal forcing, for example, often arises in childhood diseases due to cycles induced by the school year.

4. Conclusion. We consider a two-group SImodel in which net immigration from a metapopulation into the core group depends on the status of the epidemic in the core group. Two different functional dependencies of a general state-dependent recruitment function $g(S, I)=g(\xi)=a \exp \left(-b \xi^{\gamma}\right)$ were investigated: (i) $g=g(\tilde{\eta})$ and (ii) $g=g(I)$. In case (i), if the recruitment is a decreasing function of the fraction of infecteds, $g(\eta)$, the endemic equilibrium can be destabilized. Furthermore, the effect of consistent over(under)-estimation of the disease prevalence lowers(raises) the size of the endemic equilibria population (see Figure 2). Similar results hold for the rate of response of the population (see Figure 1). In case (ii), if the recruitment is a function of the number of infecteds, $g(I)$, the stability of the equilibria does not change from that of constant recruitment, however, the size of the equilibrium population is effected. It would be interesting to see how these results compare to those obtained from queuing theory and stochastic balking models.

We find that a time delay in recruitment can significantly affect these results. In case (i), $g=g(\tilde{\eta}(t-\tau))$, we found that the time delay could have a stabilizing effect on the dynamics. There are significant regions in parameter space where the endemic equilibrium alternates between being stable and unstable as $\tau$ increases (see Figure 4). In case (ii), $g=g(I(t-\tau)$ ), we found that a time delay could destabilize the dynamics. Again, we found regions in parameter space where the endemic equilibrium alternates between 
(a)

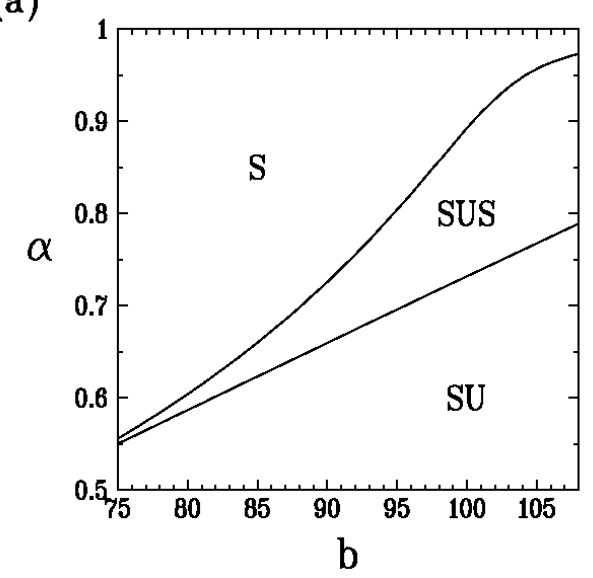

(b)

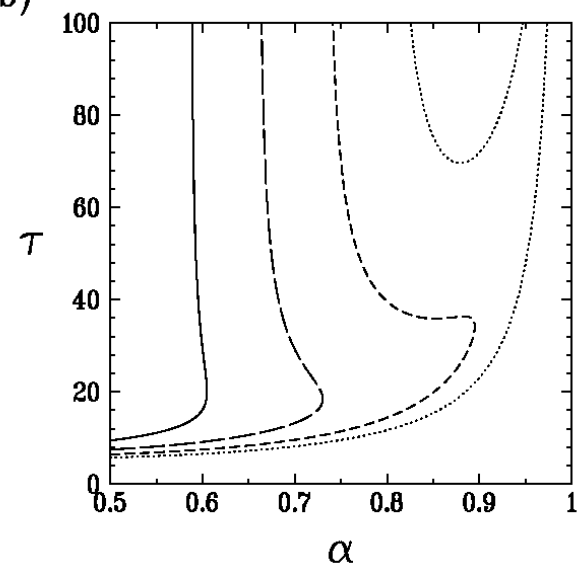

FIG. 5. (a) Stability regions of the endemic equilibrium (7) for the system (modelotd) with $g=a \exp \left(-b I(t-\tau)^{\gamma}\right)$, and $a=0.02, \mu=0.001, \chi=1.0$, and $\gamma=1.0$. To the right of the lower curve, the $S U$ region, the system is stable for small $\tau$, and unstable for $\tau>\tau_{c}$ (see Figure $5 b$ for the corresponding values of $\tau_{c}$ ). In the region between the two curves, the SUS region, the system is stable for small $\tau$, becomes unstable as $\tau$ exceeds $\tau_{1}$, and stable as $\tau$ exceeds at $\tau_{2}$ (see Figure $5 b$ for the corresponding values of $\tau$ ). To the left of both curves, the $S$ region, the system is absolutely stable. (b) Stability regions of the endemic equilibrium (7) for the system (16) with $g=$ $g(I(t-\tau))$ in the $(\tau-\alpha)$-plane for four different $b: b=80$ (solid line), $b=90$ (longdashed line), $b=100$ (short-dashed line), and $b=110$ (dotted line). The parameters are $a=0.02, \mu=0.001, \chi=1.0$, and $\gamma=1.0$. To the right of each curve, the system is stable. The boundaries between the $S$ and SUS regions are given approximately by: $\alpha>0.61$ for $b=80, \alpha>0.73$ for $b=90, \alpha>0.89$ for $b=100$, and $\alpha>0.98$ for $b=110$ (see Figure 5a). The boundary between the SU and SUS regions are approximately given by $\alpha=0.587$ for $b=80, \alpha=0.66$ for $b=90, \alpha=0.73$ for $b=100$, and $\alpha=0.81$ for $b=110$ (see Figure $5 a$ ). 
being stable and unstable as $\tau$ increases. However, these regions are small. We also found regions where, for large enough $\tau$, the equilibrium becomes unstable (see Figure 5). Indeed, this occurs in some parameter ranges even for small time delays, indicating that the system is very sensitive to $\tau$ in these regions.

We have not investigated the effects of uncertainty about the particulars of the disease or the state of the epidemic. However, there are well-developed methods for dealing with decision making in the face of uncertainty. Very generally speaking, uncertainty tends to encourage cautiousness. Hence, it seems plausible that uncertainty will tend to reduce the immigration even further, resulting in even smaller endemic equilibrium populations.

We have investigated the consequences of state-dependent net immigration in only the simplest of epidemiological models. A key assumption is that the contact rate, $c$, is constant. Since we find that the size of the population can change by over an order of magnitude, it may be appropriate, in future modelling efforts, to consider a contact rate that depends on the size of the population. We have also made many other simplifying assumptions. It may be appropriate, in specific scenarios, to generalize the model further, by, say, incorporating long and variable incubation and infectivity periods, different vaccination scenarios, more general force of infection terms which take into account decreased mobility of infecteds, and so on. Furthermore, as it was shown in Hadeler and Castillo-Chavez (1995) the size of the noncore group can play an important role. Further detail will undoubtedly complicate the analysis considerably.

Acknowledgments. We are grateful to Fred Brauer and Jorge Velasco-Hernandez for helpful discussions. This research was partially supported by NSF and NSA grants to Castillo-Chavez. This work began several years ago and was recently revised while Castillo-Chavez was visting the IMA at the University of Minnesota Minneapolis.

\section{APPENDIX}

A. Proof of global stability of the disease-free equilibrium. In this appendix, the proof of global stability of the disease-free equilibrium of the systems (5) and (16) is given. First we consider system (5). Adding the two equations in Eqs. (5), one gets $\dot{N}=g(\xi)-\mu N-\sigma I$, where $N=S+I, S \geq 0, I \geq 0$. Since $g(\xi)$, where (i) $\xi=\tilde{\eta}$ or (ii) $\xi=I$, is a decreasing function, $\dot{N}<a-\mu N$. Thus, in the limit as $t \rightarrow \infty$, both $N$ and $S$ are bounded above by $a / \mu$. From the second equation in (5), $\dot{I}<0$ for $1 \leq \alpha$. Thus $\bar{I}_{0} \geq 0$. To show that, in fact, $\bar{I}_{0}=0$, we suppose $\bar{I}_{0}>0$ and show that this leads to a contradiction. From the second equation of (5), we have 


$$
\dot{I}=-((\alpha-1) S+\alpha I) \frac{I}{S+I} \leq-\alpha I \frac{I}{S+I} \leq-\alpha \bar{I}_{0} \frac{\bar{I}_{0}}{\bar{S}_{0}+\bar{I}_{0}}<0 .
$$

Hence, in the limit as $t \rightarrow \infty, I(t)=I(0)+\int_{0}^{t} \dot{I}(r) d r \rightarrow-\infty$ which contradicts the supposition that $\bar{I}_{0}>0$ so that we must have $\bar{I}_{0}=0$. Further, since $g_{0}=a$, integrating the first equation in (5), in the limit as $t \rightarrow \infty$, one gets that $\bar{S}_{0}=a / \mu$. Since this analysis applies equally well for $g(\xi(t-\tau))$, for all $\tau>0$, it follows that the disease-free equilibrium $\left(\bar{S}_{0}, \bar{I}_{0}\right)=(a / \mu, 0)$ is globally stable if $1 \geq \alpha$ for both (model0td) and (model0dim), for both (i) $\xi=\tilde{\eta}$ or (ii) $\xi=I$.

\section{B. Linear stability analysis of the time-delayed system.}

Case (i). In this appendix, we outline the linear stability analysis of the endemic equilibrium of system (16) with $g=g(\eta(t-\tau))$. The local stability of the endemic equilibrium of (16) is determined by the sign of the real part of the eigenvalues, $\lambda=\lambda(\tau)$, of the transcendental characteristic equation (17), which changes as $\tau$ increases. To find the $\tau$ 's where changes in stability occur, we closely follow the analysis of MacDonald (1989) (and references therein), where the reader is referred to for details. It has been show that stability changes occur only at purely imaginary $\lambda$. For case (i), substituting $\lambda=i \omega$ into Eq. (17) one gets

$$
e^{-i \omega \tau}=\frac{A}{C}+\frac{i}{C}\left(\omega-\frac{B}{\omega}\right) .
$$

Plotting the two sides of (19) in the complex plane, one sees directly that if $A>C$, there are no solutions to (19). Consequently, for $A>C$, there will be no stability changes due to the time delay - equilibria that are stable for $\tau=0$ will be stable for all $\tau$. The equilibrium is said to be absolutely stable (Brauer (1987). However, if $A<C$, there are solutions, and changes in stability may occur. For $\tau=0$, if $A<C$, the equilibrium is unstable. For finite $\tau$, the system may be stabilized. We now find the ranges of $\tau$ where this occurs.

Consider first the interval where $0<\omega \tau<2 \pi$, (when the RHS of Eq. (19), the unit circle, is traced out for the first time). Eq. (19) now has two solutions, $\theta_{1}=\omega_{1} \tau_{1}$ and $\theta_{2}=\omega_{2} \tau_{2}$, where $0<\theta_{1}<\pi / 2$, and $3 \pi / 2<\theta_{2}<2 \pi$. Equating the real and imaginary parts of Eq. (19) and solving for $\omega$ one gets

$$
\omega_{ \pm}=\frac{1}{2}\left( \pm \sqrt{C^{2}-A^{2}}+\sqrt{C^{2}-A^{2}+4 B}\right),
$$

with $\omega_{2}>\omega_{1}$ (by inspection of plots of Eq. (19). Furthermore, $(d \operatorname{Re}(\lambda) /$ $d \tau)\left.\right|_{\lambda=i \omega_{1}}<0$, and $\left.(d \operatorname{Re}(\lambda) / d \tau)\right|_{i \omega_{2}}>0$. Thus, if $\tau_{1}<\tau_{2}$, an equilibrium that is unstable when $\tau<\tau_{1}$ becomes stable when $\tau>\tau_{1}$, and then, as $\tau$ exceeds $\tau_{2}$, becomes unstable again provided that at $\tau=0$ there is one pair of roots with positive real parts, all other roots have negative real parts. 
This will be the case if $(C-A)^{2}-4 B<0$. Thus, in the parameter range where $A<C,(C-A)^{2}-4 B<0$, and $\tau_{1}<\tau_{2}$, for $\tau_{1}<\tau<\tau_{2}$, the system is stable. From (19) we have

$$
\tau_{1}=\frac{\theta_{1}}{\omega_{1}} \quad, \quad \tau_{2}=\frac{\theta_{2}}{\omega_{2}},
$$

where $\theta_{1}=\arccos (A / C)$, and $\theta_{2}=2 \pi-\theta_{1}$. Thus, whether or not $\tau_{1}<\tau_{2}$ depends on the parameters $b, \alpha, \sigma, \chi$, and $\gamma$. Numerically, we find that the parameter range where $(C-A)^{2}-4 B<0$ contains the parameter range for which $\tau_{1}<\tau_{2}$. In the interval where $2 \pi<\omega \tau<4 \pi$, when the unit circle (RHS of (19)) is traced out for the second time, there are again two points where there may be stability changes, namely $\omega_{1} \tau_{1}^{(1)}=\theta_{1}+2 \pi$ and $\omega_{2} \tau_{2}^{(1)}=$ $\theta_{2}+2 \pi$. Again, if $\tau_{1}^{(1)}<\tau_{2}^{(1)}$, for $\tau_{1}^{(1)}<\tau<\tau_{2}^{(1)}$, the system will be stable. These alternating regions of stability and instability, as $\tau$ increases, will continue so long as $\tau_{1}^{(n)}<\tau_{2}^{(n)}$, where $\tau_{1}^{(n)}=\left(\theta_{1}+2 \pi n\right) / \omega_{1}$, etc., with $n$ a natural number. Since as $\tau$ increases, the stability of the equilibrium alternates between Unstable and Stable, these regions are denoted USU.

Case (ii). Proceeding as in case (i), we substitute $\lambda=i \omega$ into the characteristic equation (17). One gets

$$
e^{-i \omega \tau}=\frac{1}{D}\left(\omega^{2}-B\right)+i \omega \frac{A}{D} .
$$

This is precisely the equation discussed by MacDonald (1989), pp. 89-90. Briefly, equating the real and imaginary parts of (22), one finds that

$$
\omega^{2}=\frac{1}{2}\left(2 B-A^{2} \pm \sqrt{\left(A^{2}-2 B\right)^{2}-4\left(B^{2}-D^{2}\right)}\right) .
$$

For $0<\omega \tau<2 \pi$, one finds the following cases: Case (a) $B<D$ : Only the larger of the two $\omega$ 's in (23), $\omega_{+}$, will be real and positive. Since $\left.(d \operatorname{Re}(\lambda) / d \tau)\right|_{i \omega_{+}}>0$, if $\tau<\tau_{c}=\arccos \left(\left(\omega_{+}^{2}-B\right) / D\right) / \omega_{+}$, the equilibrium is stable, however if $\tau>\tau_{c}$, it is unstable. If $D^{2}>A^{2} B$ then $0<\omega_{+} \tau<$ $\pi / 2$, whereas if $D^{2}<A^{2} B$, one has $\pi / 2<\omega_{+} \tau<\pi$. Since the equilibrium is stable at $\tau=0$ and then becomes unstable as $\tau$ exceeds $\tau_{c}$, this region is denoted $S U$. Case (b) $B>D$ : If the radical in (23) is positive, there will be two positive real roots with $\omega_{1}>\omega_{2}$, provided that $2 B>A^{2}$. Since $\left.(d \operatorname{Re}(\lambda) / d \tau)\right|_{i \omega_{1}}>0$, and $\left.(d \operatorname{Re}(\lambda) / d \tau)\right|_{i \omega_{2}}<0$, the system will become unstable at $\tau_{1}$, as $\tau$ increases, and stable again at $\tau_{2}$, provided that $\tau_{1}<$ $\tau_{2}$. As $\tau$ increases, the stability changes will continue to alternate until $\tau_{1}^{(n)}>\tau_{2}^{(n)}$, where $\tau_{1}^{(n)}=\left(\theta_{1}+2 \pi n\right) / \omega_{1}$. Numerically, we have found only regions with one alternation. Since as $\tau$ increases, the equilibrium alternates between being stable and unstable, this region is denoted $S U S$. Case (c) $B>D$ : If the radical in (23) is negative, or if $2 B<A^{2}$, there will be no real positive solutions to (23), and, hence, there will be no stability change. 


\section{REFERENCES}

Blythe, S.P., F. Brauer and C. CAstillo-Chavez Demographic Recruitment in Sexually Transmitted Disease Model. Proc. First World Congress on Computational Medicine, Public Health and Biotechnology Part II Austin, TX, 1994. Ed. Matthew Witten. Series in Mathematical Biology and Medicine 5, 1438-1457, 1997.

Blythe, S.P., F. Brauer, . Castillo-Chavez and J. X. Velasco-Hernandez Models for sexually transmitted diseases with recruitment. Biometrics Unit Technical Report BU-1193-M, Cornell University, 1993a.

Blythe, S.P., K. Cooke And C. Castillo-Chavez Autonomous risk-behavior change, and non-linear incidence rate, in models of sexually transmitted diseases. Biometrics Unit Technical Report BU-1048-M, Cornell University, 1993b.

Brauer, F. Absolute stability in delay equations. J. Diff. Equations 69, 185-191, 1987.

BrAuer, F. Basic Ideas of mathematical epidemiology. This volume.

Brauer, F. Extensions of the basic models. This volume.

Brauer, F., C. Castillo-Chavez and J.X. Velasco-Hernandez Recruitment into Core Group and its effect on the spread of a sexually transmitted disease. (1998). Advances in Mathematical Population Dynamics - Molecules, Cells, and Man (O. Arino, D. Axelrod, M. Kimmel, (eds), World Scientific Press, 477 - 486, 1998.

Brauer, F., C. Castillo-Chavez Mathematical Models in Population Biology and Epidemiology, Texts in Applied Mathematics, Volume 40. Springer-Verlag, New York, (2001), 416 pages.

Busenberg, S. ANd C. Castillo-Chavez A general solution of the problem of mixing subpopulations, and its applications to risk- and age-structured epidemic models for the spread of AIDS. IMA J. Math. Applied in Med. and Biol. 8, 1-29, 1991.

Castillo-Chavez, C. AND S. Busenberg On the solution of the two-sex mixing problem. In Proceedings of the international conference on differential equations and applications to biology and population dynamics, Busenberg, S., Martelli, M. (eds.). Lect. Notes in Biomath., Vol. 92, pp. 80-98) Berlin Heidelberg New York, Springer 1991.

BusenBerg, S. AND K.L. CoOKE Vertically transmitted diseases: models and dynamics. Berlin New York: Springer 1993.

Castillo-Chavez. C., K. Cooke, W. Huang and S.A. Levin On the role of long incubation periods in the dynamics of acquired immunodeficiency syndrome (AIDS), Part I: Single population models. J. Math. Biol. 27, 373-398, 1989a.

Castillo-Chavez. C., K. Cooke, W. Huang and S.A. Levin The role of long incubation periods in the dynamics HIV/AIDS. Applied Math. Letters 2, 327-331, $1989 \mathrm{~b}$.

Castillo-Chavez. C., K. Cooke, W. Huang and S.A. Levin The role of long periods of infectiousness in the dynamics of acquired immunodeficiency syndrome (AIDS). In Mathematical approaches to resource management and epidemiology, CastilloChavez, C., Levin, S.A., Shoemaker, C. (eds.). Lect. Notes in Biomath., pp. 177-189, New York, Springer 1989c.

Castillo-Chavez. C., K. Cooke, W. Huang and S.A. Levin On the role of long incubation periods in the dynamics of HIV/AIDS. Part 2: Multiple group models. In Mathematical and statistical approaches to AIDS epidemiology, C. CastilloChavez (ed.), Lect. Notes in Biomath., pp. 200-217, New York, Springer 1989d.

Centers for Disease Control Self-reported behavioral change among gay and bisexual men. San Fransicsco. MMWR 34, 613-615 (1985).

Clark, C.W. Mathematical Bioeconomics. New York: John Wiley \& Sons 1990.

Evans, B.A., K.A. McLean And S.G. Dawson, et al. Trends in sexual behaviour and risk factors for HIV infection among homosexual men, 1984-1987. Br. Med. J. 198, 215-218, 1989.

Ewald, P.W. Evolution of Virulence. Scientific American 268, 86-93, 1993. 
FineBERG, H.F. Education to prevent AIDS: prospects and obstacles. Science 239, $592-596,1988$, and references therein.

Griensven, van G.J.P., E.M.M. de Vroome, J. Goudsmit and R.A. Coutino. Changes in sexual behavior and the fall in incidence of HIV infection among homosexual men. Br. Med. J. 298, 298-221, 1989.

Hadeler, K.P. And C. Castillo-Chavez A Core Group Model for Disease Transmission. Math Biosci. 128, 41-55, 1995.

Hethсоте, H.W. Three basic epidemiological models. In Applied Mathematical Ecology, Hallam, T., Gross, L., Levin, S.A. (eds.). Biomath., Vol.18, pp. 119-144, New York, Springer 1989.

Heтнсоте, H.W., The mathematics of infectious diseases , SIAM Review, 42, 4, pp. $599-653$.

Hethcote, H.W. AND Yorke, J.A. Gonorrhea: Transmisison dynamics and control. Lecture notes in Biomath., Vol. 56, Berlin, Springer 1984.

Holling, C.S. Some characteristics of simple types of predation and parasitism. Can. Ent. 91, 385-393, 1959.

Hoover, D.R., A. Munoz, V. Carey, N. Odaka, J.M.G. Taylor, J.S. Chmiel, J. Armstrong and S.H. Vermund The unseen sample in cohort studies estimation of its size and effect. Statistics in Medicine 10, 1993-2003, 1991.

HSIEH, Y. AND K. COOKE Behaviour change and treatment of core groups: its effect on the spread of HIV/AIDS. IMA J. of Math. Applied to Medicine and Biology 17, $213-241,2000$.

Huang, W. and C. Castillo-Chavez Age-structured Core Group Model and its impact on STD dynamics. This volume.

Huang, W, K. L. Cooke, and C. Castillo-Chavez, Stability and bifurcation for a multiple-group model for the dynamics of HIV/AIDS transmission, SIAM J. Appl. Math., 52, pp. 835-854, 1992.

Hyman J. M. AND E. A. Stanley, Using mathematical models to understand the AIDS epidemic, Math. Biosci., 90, pp. 415-473, 1988.

Hyman J. M. AND E. A. STANLEY, The effect of social mixing patterns on the spread of AIDS. In Mathematical Approaches to Problems in Resource Management and Epidemiology, C. Castillo-Chavez, S. A. Levin, and C. Shoemaker, eds., Lecture Notes in Biomathematics, vol. 81, Springer-Verlag, New York, 1989.

Hyman, J. M., LI, J. And E. A. Stanley, Threshold conditions for the spread of HIV infection in age-structured populations of homosexual men, J. Theor. Biol., 166, pp. $9-31,1994$.

Jacquez J. A., C. P. Simon, J.S. Koopman, L. Sattenspiel, and T. Perry, Modeling and analyzing HIV transmission: The effect of contact patterns, Math. Biosci., 92, pp. $119-199,1988$.

MACDonald, N. Biological delay systems: linear stability theory. Cambridge: Cambridge University Press 1989.

MARTIN, J.L. The impact of AIDS in gay male sexual behavior patterns in New York City. Am. J. of Public Health 298, 578-581, 1987.

MCClAM, E. "Syphilis Outbreak alarms officials", Ithaca Journal, Section A., page 10A, February 23, 2001.

McKusick, L., W. Horstman And T.L. Coates AIDS and sexual behavior reported by gay men in San Fransisco. Public Health Reports 75, 493-496, 1985a.

McKusick, L., J.A. Wiley, T.L. Coates, R. Stall, B. Saika, S. Morin, K. Charles, W. Horstman AND M.A. ConANT Reported changes in the sexual behavior of men at risk for AIDS. San Fransisco, 1983-1984: the AIDS behavioral research project, Public Health Reports 100, 622-629, 1985b.

Murray, J.D. Mathematical Biology. (Biomath., Vol. 19). Springer-Verlag, Berlin Heidelberg New York, 1990.

Palmer, J.S., C. Castillo-Chavez and S. P. Blythe State-dependent mixing and state-dependent contact rates in epidemiological models. Biometrics Unit Technical Report BU-1122-M, Cornell University, 1991. 
Perez-Stable, E. Cuba's response to the HIV epidemic. Americ. J. Public Health 8, $5,563-567,1991$.

Pinder, J.E., J.G. Wiener And M.H. Smith The Weibull distribution: a new method of summarizing survivorship data. Ecology 59, 1, 175-179, 1978.

Sattenspiel, L. Population structure and the spread of disease. American Journal of Physical Anthropology 73, 251-265, 1987a.

SATTENSPIEL, L. Epidemics in nonrandomly mixing populations. Human Biology 59, 411-438, 1987b.

SATtEnspiel, L. AND C. Simon The spread and persitence of infectious diseases in structured populations. Math. Bios. 90, 341-366, 1988.

SCALia-Tomba, G. The effect of structural behavior change on the spread of HIV. Math. Bios. 107, 547-556, 1991.

Thieme, H.R. and C. Castillo-Chavez On the role of variable infectivity in the dynamics of the human immunodeficiency virus epidemic. In Mathematical and statistical approaches to AIDS epidemiology, Castillo-Chavez, C. (ed.). Lecture notes in Biomath., Vol. 83, pp. 157-176. Berlin: Springer 1989.

Thieme, H.R. And C. Castillo-Chavez How May Infection-Age-Dependent Infectivity Affect the Dynamics of HIV/AIDS. (1993). SIAM J. Applied Math. 53, No. 5, pp. 1447-79.

Velasco-Hernandez, J. X., F. Brauer and C. Castillo-Chavez Effects of Treatment and Prevalence-dependent Recruitment on the Dynamics of a fatal disease. IMA Journal of Math. Medicine and Biology, 13 3, 175-192, 1996.

Velasco-Hernandez J.X. AND Y. HSIEH Modelling the effect of treatment and behavioral change in HIV transmission dynamics. J. Math Biol. 32, 233-249, 1994. 